\title{
Antimicrobial Activity of Extract from the Abdominal Gland of African Weaver Ants (Oecophylla longinoda) on the Microorganisms Isolated from Cocoa Pods
}

\section{Oladunmoye Muftau $\mathrm{K}^{1}$, Isijola Samuel $\mathbf{O}^{1}$ and Oladosu Tolulope $\mathbf{O}^{2 *}$}

${ }^{1}$ Department of Microbiology, Federal University of Technology, Akure, Ondo State, Nigeria ${ }^{2}$ Department of Biological Sciences, Bells University of Technology, Ota, Ogun State, Nigeria *Corresponding Author: Oladosu Tolulope O, Department of Biological Sciences, Bells University of Technology, Ota, Ogun State, Nigeria.

Received: January 22, 2018; Published: February 21, 2018

DOI: $10.31080 /$ ASMI.2018.01.0028

\section{Abstract}

This research was conducted to investigate into the antimicrobial potency of the abdominal gland extract of African Weaver Ant (AWA) against microorganisms isolated from Cocoa pods. Six (6) fresh samples of both infected and uninfected Cocoa pods gotten from a village farm in Ibule-soro, Ondo State were transferred to the laboratory under aseptic conditions. One gram from the pod of each sample was subjected to microbial analysis for isolation and the antimicrobial effects of the AWA abdominal extract was subsequently evaluated. Selected conventional antibiotics were used as controls. Bacterial isolates obtained were Bacillus subtilis, Pseudomonas aeruginosa, Serratia fonticola, Proteus penneri, Enterobacter cloacae, Citrobacter freundii, Edwardsiella tarda, Klebsiella oxytoca and Proteus mirabilis, while fungal isolates were Aspergillus aculeatinus, Alternaria sp., Chloridium chlamydosporis, Aspergillus niger, Phytophthora palmivora, Aspergillus flavus, Aspergillus tamari, Fusarium sp., Penicillium chrysogenum, Penicillium camemberti, Saccharomyces cerevisiae, Saccharomycodes ludwigii. Four of all the bacterial isolates (Bacillus subtilis, Edwardsiella tarda, Proteus penneri, Proteus mirabilis) used for this study exhibited high levels of sensitivity to the abdominal gland of African weaver ants with the following zones of inhibition: $19 \mathrm{~mm}, 19 \mathrm{~mm}, 20 \mathrm{~mm}$ and $21 \mathrm{~mm}$ respectively. Similarly, fungal isolates were observed to be sensitive to the Oecophylla longinoda abdominal extract, with Aspergillus flavus having the highest zone of inhibition (14.89 mm). This research reveals the potentials of abdominal extracts from African weaver ants (Oecophylla longinoda) in antimicrobial capacities against most aetiological agents of Cocoa diseases.

Keywords: Cocoa; Oecophylla Longinoda; Antibacterial; Antifungal

\section{Introduction}

Cacao, a species in the genus Theobroma, is the most economically important species in this genus (Acebo-Guerrero 2012). It is an upright, evergreen tree that grows up to 15 metres, but it is usually kept below 5 metres to make for easy harvesting. The tree has a polymorphic characteristic - its seedling grows to between 1 to $2 \mathrm{~m}$ in height as a single stem after which it separates into the jorquette (a whorl of lateral-growing 3-5 branches). Two to three years after planting, flowers begin to grow from pads or cushions beneath the bark. Under optimal conditions these pads will continue to produce flowers for 60 to 100 years. Some self-incompatible cacao varieties usually require cross-pollination, while others that are completely self-compatible do not need to be cross-pollinated. The cacao fruit (pod) is cut from the tree without impairing the flower pad below the surface because impaired flower pads will no longer bear fruit. The pods are harvested for the seeds (beans) usually found inside them. Each pod contains about 30 to 60 beans, these are enclosed with white mucilage that is high in sugar content. Pods are picked at maturity for their primary use which is the production of chocolate. Cacao can be grown only within the tropical belt [1]. Ivory Coast on the west coast of Africa, is reputed to be the largest cacao-producing region where $68 \%$ of the world's cacao is being harvested. Theobroma cacao is believed to have its origin in The Amazon Basin which makes up only a portion of the $15 \%$ of world production from the Americas [2]. Phytophthora pod rot (PPR or black pod) and Cocoa swollen shoot virus (CSSV) are two diseases considered to be highly important in the production of cocoa, these pathogens have consequently received great attention in Nigeria. Phytophthora pod rot caused by P. palmivora and $P$. megakarya, was acclaimed the most important yield-limiting factor in the Nigerian cocoa industry, with total annual losses estimated at up to 30 to $35 \%$ of the nation's crop [3], while $30-90 \%$ of the total global crop loss is caused by this disease [4]. However, severity of this disease varies depending on climatic factors like rainfall and humidity and the variety of cocoa concerned. Seedling diseases, cankers of stems, trunks and crowns of cocoa trees could be resultant from Phytophthora spp. infestations. The sources of inoculum for PPR are soil, ant tents, infected flower cushions, dry pods on the cocoa trees or ground, debris formed from heaping of cocoa pod husks. Spread from infected pods is by water splash and drips during rains, living vectors, wind-blown sprays and wounds or non-intact surfaces on pods usually caused by knives, rodents

Cocoa is a very important cash crop but the advent of the black pod disease caused by Phytophthora megakarya has greatly reduced the revenue generated by farmers. P. megakarya black pod is the single most important fungal disease of cocoa which has 
been described by some farmers as a strange disease [8], this is due to the extreme virulence and the heavy losses associated with the pathogen. Many highly or extremely toxic pesticides (Class I) are being used in developing countries [9]. Spilling of pesticides over the back of the operator owing to a faulty locking cap of the container is a major cause of poisoning when using knapsack or trombone sprayer [10].

Various species of Weaver ants (Oecophylla spp.) have been used for pest management in tropical agro-ecosystems across Australia Asia and Africa [11,12]. They have proven to be highly effective biological control agents being capable of controlling over 50 species of insect pests in at least 12 different tropical crops [11,13]. The use of conventional synthetic insecticide control was substituted with weaver ants and this led to increased net incomes of more than $70 \%$ in Australian cashew and mango plantations $[14,15]$. The peculiarities of extremely organized predatory behaviour, ability to forage the entire areas occupied by colonies and their potential to expand into new areas are responsible for the success of weaver ants in killing or driving out many potential pests $[16,17]$. In the year 1991, Dejean demonstrated the predatory efficiency of the African weaver ant Oecophylla longinoda and estimated that a colony of 12 nests captured approximately 45,000 prey individuals per year [18].

Oecophylla worker ants are utilised directly as a commercial product, as they are reported to be a source of traditional Chinese and Indian medicines against arthritis and some other abnorma health conditions $[19,20]$. Cocoa as a cash crop has over the years been faced with various diseases which have resulted into reduced yield and decrease in the total revenue of cocoa farmers. Consequently, some cocoa farms have been abandoned while other farmers have switched over to the cultivation of other crops [8]. This research is therefore designed to investigate into the antimicrobia properties of extracts from the abdominal gland of 0 . longinoda in the prevention and treatment of diseases affecting Cocoa. This research aims to isolate and characterize the microorganisms from the infected and uninfected Cocoa pods, to evaluate the antimicrobial properties of extract from the abdominal gland of the African Weaver Ants (Oecophylla longinoda) using conventional antibacterial and antifungal agents as a control study group to the research.

\section{Materials and Methods}

Collection of Samples

Six samples, (three Infected and three uninfected) Cocoa samples were collected from a selected farm in Ibule - soro, Ondo State, aseptically into already sterilized containers and were transported to the Microbiology research laboratory of the Federal University of Technology, Akure for microbial analysis.

\section{Sterilization of Materials and Media}

All glassware used were properly washed with detergent and rinsed thoroughly with water, allowed to dry and sterilized in the hot air oven at $160^{\circ} \mathrm{C}$ for 2 hours. The culture media used were prepared in the conical flasks following the manufacturer's standard. Test tubes used were plugged with cotton wool and wrapped with aluminium foil. Inoculating loops and needles were sterilized using a Bunsen burner till red heat was acquired. Work surfaces were swabbed with cotton wool soaked in $70 \%$ alcohol before and after each analysis. Hands were washed with an antiseptic soap before and after each experiment to avoid contamination and infection.

\section{Isolation of Bacteria from Cocoa pods}

The surface of the pod was washed properly with sterile distilled water [21-23]. One gram (1g) of the Cocoa pod was cut from each of the infected and uninfected pods and ground using mortar and pestle. This was transferred into the first test tube (containing
$10 \mathrm{ml}$ of sterile distilled water) and diluted serially in a stepwise manner (in test tubes with $9 \mathrm{ml}$ of sterile distilled water) to $10^{-7}$ dilution test tube. One millilitre $(1.0 \mathrm{ml})$ each of the dilution $10^{-5}$, $10^{-6}$ and $10^{-7}$ was plated on Nutrient agar, the plates were inverted and incubated at $37^{\circ} \mathrm{C}$ for $24 \mathrm{~h}$ after which the plates were examined for growth.

\section{Isolation and Characterization of Bacterial Isolates}

The bacterial growth on nutrient agar was sub-cultured by streaking until pure colonies were obtained according to the conventional procedure as highlighted by [24]. Cultural characteristics of the discrete bacterial colonies such as colour, shape, pigmentation and opacity on nutrient agar were observed and noted. This was followed by characterization of the isolates [25] that includes microscopic examination for cell morphology, gram reaction and biochemical tests on $24 \mathrm{~h}$ old cultures. Discrete bacterial colonies were cultured on double strength nutrient agar slant and incubated at $37^{\circ} \mathrm{C}$ for $24 \mathrm{~h}$, growth was observed and the slants were stored in the refrigerator for preservation of bacterial isolates.

Morphological and Biochemical Characterization of Bacterial Isolates

Different tests were carried out on isolates which include; Gram reaction, Urease, Oxidase, Voges Proskauer, Catalase test [24], Indole production, Motility and Citrate utilization test [25]. Bacterial isolates were identified using standard cultural, morphological, and biochemical characteristics as described by Cheesbrough [25].

Hydrogen Sulphide and Gas Production on Triple Sugar Iron (TSI) agar

The medium was prepared according to the manufacturer's specification. Five milliliter $(5 \mathrm{ml})$ was dispensed into sterilized test tube and autoclaved at $121^{\circ} \mathrm{C}$ for 15 minutes. The medium was allowed to solidify in a slanting position to give a butt 25 $30 \mathrm{~mm}$ deep and a slope $20-25 \mathrm{~mm}$ long. A straight wire was used to inoculate TSI agar slope medium with the inoculum of the test isolate by stabbing the butt and then streaking the surface of the slope in a zig-zag pattern and incubate at $37^{\circ} \mathrm{C}$ for $24 \mathrm{~h}$. TSI reactions are based on the fermentation of lactose and glucose (dextrose) and the production of hydrogen sulphide. Presence of cracks in the tube indicates gas production and black colouration indicates hydrogen sulphide production [25].

\section{McFarland Turbidity Standard}

Standardization of inoculum was done using WPA colorimeter. Five milliliter ( $5 \mathrm{ml}$ ) of sterile normal saline in test tubes of equal sizes was inoculated with 18 - $24 \mathrm{~h}$ old culture. An uninoculated test tube serves as a control to calibrate the colorimeter. The suspension of each isolate was adjusted using 0.5 McFarland standard at $590 \mathrm{~nm}$ to obtain about $10^{6}-10^{8} \mathrm{cfu} / \mathrm{ml}$ for the isolates

\section{Antibacterial Susceptibility Test}

Antibiotic resistance of bacteria was determined by the single disc diffusion method with the use of Mueller-Hinton agar, according to the Kirby-Bauer's method. The medium used was MuellerHinton agar (MHA) in petri dishes. The surface of the agar was aseptically seeded with a standardized saline suspension of the isolate using sterile swab sticks. Antibiotic discs consisting Pefloxacin $(10 \mu \mathrm{g})$, Gentamycin $(10 \mu \mathrm{g})$, Ampiclox $(30 \mu \mathrm{g})$, Zinacef $(20 \mu \mathrm{g})$, Amoxicillin $(30 \mu \mathrm{g})$, Rocephin $(25 \mu \mathrm{g})$, Ciprofloxacin (10 $\mu \mathrm{g})$, Streptomycin $(30 \mu \mathrm{g})$, Septrin $(30 \mu \mathrm{g})$, Erythromycin $(10 \mu \mathrm{g})$, Chloramphenicol $(30 \mu \mathrm{g})$, Sparfloxacin $(10 \mu \mathrm{g})$, Tarivid $(30 \mu \mathrm{g})$, Augmentin $(25 \mu \mathrm{g})$ were aseptically placed on the surface of the MHA and the plates were incubated at $37^{\circ} \mathrm{C}$ for $18-24$ hours. The diameters of the zones of inhibition were then measured using a transparent meter rule to the nearest millimeter. 


\section{Antibacterial assay of the Ants}

The abdomen of the Oecophylla longinoda ant was cut and crushed using mortar and pestle and then homogenized with Dimethyl sulfoxide (DMSO) under aseptic conditions and diluted into three different concentrations in such a way that $100 \mu$ l homogenate contained glands of 3,6 and 12 the assay was conducted using agar well diffusion method [26].

\section{Isolation of Fungi from Cocoa Pods}

The surface of the pod was washed properly with sterile distilled water [21-23]. One gram (1g) of the Cocoa pod was cut from each of the infected and uninfected pods and ground using mortar and pestle. This was transferred into the first test tube (containing 10 $\mathrm{ml}$ of sterile distilled water) and diluted serially in a stepwise manner (in test tubes with $9 \mathrm{ml}$ of sterile distilled water) to $10^{-7}$ dilution test tube. One millilitre $(1.0 \mathrm{ml})$ each of the dilution $10^{-3}, 10^{-4}$ and $10^{-5}$ was plated on Potato dextrose agar, the plates were incubated aerobically at $25 \pm 2^{\circ} \mathrm{C}$ for $3-7$ days after which the plates were examined for growth.

Fungal mycelia were teased with sterile inoculating needle. With two drops of Lactophenol - cotton blue dye were added to the sample on a clean glass slide and covered with slip and then viewed under the light microscope using medium and high power objective lenses. The Fungal characterization was based on micro morphological features which include the following; nature of conidia shape, sporangiophore, arthrospores, spore head, rhizoid and hyphae (septate or non-septate). Isolated fungal colonies were identified for both microscopic and macroscopic examinations [24].

\section{Antifungal Susceptibility Test of Fungi isolated from Cocoa pods}

This test was used to determine the sensitivity of fungal isolates to selected antifungal agents. Four commercial antifungal agents (Griseofulvin, Ketoconazole, Itraconazole and Clotrimazole) were used in this assay. These were reconstituted to a concentration of $40 \mathrm{mg} / \mathrm{ml}$ each and agar well method was adopted for the test. The medium used was Mueller-Hinton agar. The surface of the agar was aseptically seeded with a standardized saline suspension of the isolate using sterile swab sticks and the plates were incubated at $37^{\circ} \mathrm{C}$ for 24 hours. The diameter of the zone of inhibition was then measured using a transparent meter rule to the nearest millimeter.

\section{Antifungal assay of the Ants}

The abdomen of the Oecophylla longinoda ant was cut and crushed using mortar and pestle and then homogenized with DMSO under aseptic conditions and diluted into three different concentrations in such a way that $100 \mu \mathrm{l}$ homogenate contained glands of 3, 6 and 12 using agar well diffusion method [26].

\section{Results}

Bacterial load on the Cocoa Pods

Mean total bacterial counts were shown in table 1 . The result revealed there was significant difference $(p \leq 0.05)$ in the bacte- rial load of the Cocoa pods. The bacterial load on the Cocoa pods showed a range of $15.00 \pm 0.29 \times 10^{6} \mathrm{cfu} / \mathrm{g}$ to $20.00 \pm 1.15 \times 10^{6}$ $\mathrm{cfu} / \mathrm{g}$ in infected pods and $5.00 \pm 0.57 \times 10^{6} \mathrm{cfu} / \mathrm{g}$ to $10.00 \pm 0.98$ x $10^{6} \mathrm{cfu} / \mathrm{g}$ in uninfected pods. Bacterial load of infected sample 1 was significantly $(p \leq 0.05)$ higher than other samples.

\begin{tabular}{|c|c|c|}
\hline Samples & $\begin{array}{c}\text { Infected Cocoa pods } \\
\text { (cfu/g } \times \mathbf{1 0}^{6} \text { ) }\end{array}$ & $\begin{array}{c}\text { Uninfected Cocoa pods } \\
\text { (cfu/g } \mathbf{~} \mathbf{1 0}^{\mathbf{6}} \text { ) }\end{array}$ \\
\hline 1 & $20.00 \pm 1.15^{\mathrm{b}}$ & $10.00 \pm 0.98^{\mathrm{b}}$ \\
\hline 2 & $15.00 \pm 0.29^{\mathrm{a}}$ & $7.00 \pm 0.34^{\mathrm{a}}$ \\
\hline 3 & $19.00 \pm 0.69^{\mathrm{b}}$ & $5.00 \pm 0.57^{\mathrm{a}}$ \\
\hline
\end{tabular}

Table 1: Bacterial Load on the Cocoa Pods. Values are presented as mean $\pm \mathrm{SE}$ and values in the same column carrying same superscript are not significantly different using Duncan's new multiple range test at $\mathrm{p} \leq 0.05$

Key: SE: Standard Error; Cfu: Colony Forming Unit

Fungal load on the Cocoa Pods

Mean total fungal spore counts were shown in table 2. The result revealed there was significant difference $(\mathrm{p} \leq 0.05)$ in total fungal spores count load of the Cocoa pods. The fungal load of Cocoa pods showed a range of $2.67 \pm 0.33 \times 10^{6} \mathrm{sfu} / \mathrm{g}$ to $11.33 \pm 0.88$ $\mathrm{x} 10^{6} \mathrm{sfu} / \mathrm{g}$ in infected pods and $1.33 \pm 0.33 \times 10^{6} \mathrm{sfu} / \mathrm{g}$ to $2.33 \pm$ $0.33 \times 10^{6} \mathrm{sfu} / \mathrm{g}$ in uninfected pods. Fungal load of infected sample 1 was significantly ( $\mathrm{p} \leq 0.05)$ higher than other samples.

\begin{tabular}{|c|c|c|}
\hline Samples & $\begin{array}{c}\text { Infected } \\
\left(\mathbf{s f u} / \mathbf{g} \times \mathbf{1 0}^{3}\right)\end{array}$ & $\begin{array}{c}\text { Uninfected } \\
\left(\mathbf{s f u} / \mathbf{g} \times \mathbf{1 0}^{\mathbf{}} \text { ) }\right.\end{array}$ \\
\hline 1 & $11.33 \pm 0.88^{\mathrm{c}}$ & $1.33 \pm 0.33^{\mathrm{a}}$ \\
\hline 2 & $6.00 \pm 0.58^{\mathrm{b}}$ & $1.66 \pm 0.33^{\mathrm{a}}$ \\
\hline 3 & $2.67 \pm 0.33^{\mathrm{a}}$ & $2.33 \pm 0.33^{\mathrm{a}}$ \\
\hline
\end{tabular}

Table 2: Fungal Load on Cocoa Pods.

Values are presented as mean $\pm \mathrm{SE}$ and values in the same column carrying same superscript are not significantly different using Duncan's new multiple range test at $\mathrm{p} \leq 0.05$ Key: SE: Standard Error; Sfu: Spore Forming Unit

Morphological, Biochemical Characteristics and Identification of isolates from Cocoa Pods

Table 3 shows biochemical tests that were carried out on the bacterial isolates; Gram stain, Cell shape, motility, catalase, citrate, oxidase, indole, reaction on Triple Sugar Iron. All the bacterial isolates show different biochemical reactions and were characterized morphologically based on microscopy into cocci and bacilli. The isolates were identified as Bacillus subtilis, Pseudomonas aeruginosa, Serratia fonticola, Proteus penneri, Enterobacter cloacae, Citrobacter freundii, Edwardsiella tarda, Klebsiella oxytoca and Proteus mirabilis. 


\begin{tabular}{|c|c|c|c|c|c|c|c|c|c|c|c|c|c|c|}
\hline $\begin{array}{l}\text { Isolate } \\
\text { code }\end{array}$ & $\begin{array}{l}\text { Gram's } \\
\text { reaction/ } \\
\text { shape }\end{array}$ & $\begin{array}{c}\text { Motility } \\
\text { test }\end{array}$ & $\begin{array}{c}\text { Catalase } \\
\text { test }\end{array}$ & $\begin{array}{c}\text { Citrate } \\
\text { test }\end{array}$ & $\begin{array}{c}\text { Oxidase } \\
\text { test }\end{array}$ & $\begin{array}{c}\text { Indole } \\
\text { Test }\end{array}$ & Top & Butt & $\mathrm{H}_{2} \mathrm{~S}$ & $\begin{array}{c}\text { Gas } \\
\text { produc- } \\
\text { tion }\end{array}$ & $\begin{array}{c}\text { Methyl } \\
\text { red }\end{array}$ & $\begin{array}{l}\text { Voges- } \\
\text { Pros- } \\
\text { kaeur }\end{array}$ & $\begin{array}{c}\text { Urease } \\
\text { test }\end{array}$ & $\begin{array}{c}\text { Probable } \\
\text { Organisms }\end{array}$ \\
\hline $\mathrm{I}_{1} \mathrm{a}$ & $+/ \mathrm{rod}$ & + & + & + & - & - & $\mathrm{Y}$ & $\mathrm{R}$ & - & - & - & + & - & $\begin{array}{l}\text { Bacillus } \\
\text { subtilis }\end{array}$ \\
\hline $\mathrm{I}_{1} \mathrm{~b}$ & $-/ \mathrm{rod}$ & + & + & + & + & - & $\mathrm{R}$ & $\mathrm{R}$ & - & - & - & - & - & $\begin{array}{l}\text { Pseudomonas } \\
\text { aeruginosa }\end{array}$ \\
\hline $\mathrm{I}_{2} \mathrm{a}$ & $-/$ rod & + & + & - & - & - & $\mathrm{Y}$ & $\mathrm{Y}$ & - & + & - & + & - & $\begin{array}{c}\text { Serratia } \\
\text { fonticola }\end{array}$ \\
\hline $\mathrm{I}_{2} \mathrm{~b}$ & $+/ \mathrm{rod}$ & + & + & - & - & - & $\mathrm{R}$ & $Y$ & - & - & - & + & - & $\begin{array}{l}\text { Bacillus } \\
\text { subtilis }\end{array}$ \\
\hline $\mathrm{I}_{2} \mathrm{C}$ & $-/ \mathrm{rod}$ & + & + & - & - & - & $\mathrm{R}$ & $Y$ & - & - & + & - & + & $\begin{array}{l}\text { Proteus pen- } \\
\text { neri }\end{array}$ \\
\hline $\mathrm{I}_{3} \mathrm{a}$ & $-/ \mathrm{rod}$ & + & + & - & - & - & $\mathrm{Y}$ & $\mathrm{Y}$ & - & + & - & + & - & $\begin{array}{c}\text { Enterobacter } \\
\text { cloacae }\end{array}$ \\
\hline $\mathrm{I}_{3} \mathrm{~b}$ & $-/ \mathrm{rod}$ & + & + & - & - & - & $Y$ & $Y$ & - & + & + & + & - & $\begin{array}{l}\text { Citrobacter } \\
\text { freundii }\end{array}$ \\
\hline $\mathrm{I}_{3} \mathrm{c}$ & $-/ \operatorname{rod}$ & + & + & - & - & - & $\mathrm{R}$ & $Y$ & + & + & - & - & - & $\begin{array}{c}\text { Edwardsiella } \\
\text { tarda }\end{array}$ \\
\hline $\mathrm{I}_{3} \mathrm{~d}$ & $-/ \operatorname{rod}$ & + & + & - & - & - & $\mathrm{Y}$ & $\mathrm{Y}$ & - & + & + & + & - & $\begin{array}{l}\text { Citrobacter } \\
\text { freundii }\end{array}$ \\
\hline $\mathrm{UI}_{1} \mathrm{a}$ & $-/ \operatorname{rod}$ & + & + & + & - & - & $\mathrm{Y}$ & $Y$ & - & + & - & + & - & $\begin{array}{c}\text { Klebsiella } \\
\text { oxytoca }\end{array}$ \\
\hline $\mathrm{UI}_{1} \mathrm{~b}$ & $-/ \mathrm{rod}$ & + & + & - & - & - & $\mathrm{R}$ & $\mathrm{Y}$ & - & + & + & - & + & $\begin{array}{l}\text { Proteus pen- } \\
\text { neri }\end{array}$ \\
\hline $\mathrm{UI}_{2} \mathrm{a}$ & $-/ \operatorname{rod}$ & + & + & - & - & - & $R$ & $Y$ & + & + & + & - & + & $\begin{array}{c}\text { Proteus mira- } \\
\text { bilis }\end{array}$ \\
\hline $\mathrm{UI}_{3} \mathrm{a}$ & $-/ \operatorname{rod}$ & + & + & - & + & - & $Y$ & $\mathrm{R}$ & - & + & - & - & - & $\begin{array}{c}\text { Pseudomonas } \\
\text { aeruginosa }\end{array}$ \\
\hline
\end{tabular}

Table 3: Morphological, Biochemical Characteristics and identification of Bacteria isolated from Cocoa pods.

Key: +: Positive; -: Negative; Y: Yellow; R: Red

Identification and cultural characteristics of fungi isolated from Cocoa pods

Table 4 revealed the fungal characterization which was based on micro and macro morphological features that include the following; nature of conidia shape, sporangiophore, arthrospores, spores head, rhizoid and hyphae (septate or non-septate). Isolated fungal colonies were identified from both microscopic and macroscopic examinations and they were identified to be Aspergillus aculeatinus, Penicillium chrysogenum, Chloridium chlamydosporis, Aspergillus niger, Saccharomycodes ludwigii, Phytophthora palmivora, Aspergillus flavus, Saccharomyces cerevisiae, Aspergillus tamari, Fusarium sp., Penicillium camemberti, Alternaria species.

\begin{tabular}{|c|c|}
\hline Fungal isolates & $\begin{array}{l}\text { Morphological and microscopy } \\
\text { characterization }\end{array}$ \\
\hline Aspergillus aculeatinus & $\begin{array}{l}\text { Dark green colouration on agar plate, clavate } \\
\text { swelling, conidiophores not too long, upright, } \\
\text { conidia globose or ovoid, conidophores standing } \\
\text { inform of mortar pistle, thick with swollen head. }\end{array}$ \\
\hline $\begin{array}{l}\text { Penicillium } \\
\text { chrysogenum }\end{array}$ & $\begin{array}{l}\text { Green colonies on culture. Conidia are unicel- } \\
\text { lular round to ovoid, hyaline or pigmented. } \\
\text { Phialides grouped in brush-like cluster at the } \\
\text { end of the conidiophores. }\end{array}$ \\
\hline Penicillium camemberti & $\begin{array}{l}\text { White to pink or orange colonies. Mycelium con- } \\
\text { sists of a highly branched networkof multinucle- } \\
\text { ate septate. }\end{array}$ \\
\hline Alternaria sp. & $\begin{array}{l}\text { Colonies are fast growing, black or greyish. } \\
\text { Conidia are obclavate, sometimes ovoid or ellip- } \\
\text { soidal, often with a short conical break smooth- } \\
\text { walled or verrucose. }\end{array}$ \\
\hline $\begin{array}{l}\text { Chloridium } \\
\text { chlamydosporis }\end{array}$ & $\begin{array}{l}\text { Black on culture. Conidiophores slender and } \\
\text { swollen, lightly pigmented, simple or septate, } \\
\text { conidia subhyaline, I- celled globose or oblong. }\end{array}$ \\
\hline Aspergillus niger & $\begin{array}{l}\text { Black colouration with black conidia with white } \\
\text { lining on the culture. Conidiophores are very } \\
\text { thick and long. }\end{array}$ \\
\hline $\begin{array}{l}\text { Saccharomycodes } \\
\text { ludwigii }\end{array}$ & Cream to yellow colonies with budding cells. \\
\hline $\begin{array}{l}\text { Phytophthora palmi- } \\
\text { vora }\end{array}$ & $\begin{array}{l}\text { White colonies with uneven edges. From mi- } \\
\text { croscopic observation, there was presence of } \\
\text { hyphae hyaline, branches and non-partition. }\end{array}$ \\
\hline Aspergillus flavus & $\begin{array}{l}\text { Green colonies on culture, globose conidia. The } \\
\text { hyphae is branched and the septate conidio- } \\
\text { phore are long, rough, septate and granular. }\end{array}$ \\
\hline $\begin{array}{l}\text { Saccharomyces cere- } \\
\text { visiae }\end{array}$ & $\begin{array}{l}\text { White to cream, smooth, glaborous yeast-like } \\
\text { colonies. Large globose to ellipsoidal cells with } \\
\text { multilateral budding. }\end{array}$ \\
\hline Aspergillus tamari & $\begin{array}{l}\text { Dirty green colouration on the culture, dense } \\
\text { spotting on culture }\end{array}$ \\
\hline Fusarium culmorum & $\begin{array}{l}\text { White cotton wool-like mould growth with } \\
\text { no distinct aerial mycelium. Conidiophore are } \\
\text { short, single, lateral monophialides }\end{array}$ \\
\hline
\end{tabular}

Table 4: Morphological characteristics of fungal isolates.
Comparative distribution and occurrence of bacteria isolated from Cocoa pods

The total number of bacteria isolated from each of the samples collected were presented in table 5a, the occurrence of each bacterial isolates from both the infected and uninfected pods as well as the percentage occurrences were also presented.

\begin{tabular}{|c|c|c|c|c|c|c|c|c|}
\hline \multirow{2}{*}{$\begin{array}{c}\text { Isolates } \\
\begin{array}{c}\text { Bacterial } \\
\text { isolates }\end{array}\end{array}$} & \multicolumn{3}{|c|}{ Infected } & \multicolumn{3}{|c|}{ Uninfected } & \multirow[t]{2}{*}{ Total } & \multirow{2}{*}{$\begin{array}{c}\text { Occurrence } \\
(\%)\end{array}$} \\
\hline & A & B & C & A & B & C & & \\
\hline Bacillus subtilis & + & + & - & & - & - & 2 & 9.1 \\
\hline $\begin{array}{l}\text { Psuedomonas } \\
\text { aeruginosa }\end{array}$ & + & - & - & & - & + & 2 & 9.1 \\
\hline $\begin{array}{l}\text { Serratia } \\
\text { fonticola }\end{array}$ & & + & - & & - & - & 1 & 4.5 \\
\hline Proteus penneri & & + & + & & - & - & 2 & 9.1 \\
\hline $\begin{array}{l}\text { Enterobacter } \\
\text { cloacae }\end{array}$ & + & - & + & & - & - & 2 & 9.1 \\
\hline $\begin{array}{l}\text { Citrobacter } \\
\text { freundii }\end{array}$ & & - & + & - & - & - & 1 & 4.5 \\
\hline $\begin{array}{l}\text { Edwardsiella } \\
\text { tarda }\end{array}$ & & + & - & & + & - & 2 & 9.1 \\
\hline $\begin{array}{l}\text { Klebsiella } \\
\text { oxytoca }\end{array}$ & & - & - & + & - & + & 2 & 9.1 \\
\hline Proteus penneri & + & + & + & + & + & + & 6 & 27.3 \\
\hline Proteus mirabilis & - & + & - & - & + & - & 2 & 9.1 \\
\hline Total & 4 & 6 & 4 & 2 & 3 & 3 & 22 & 100 \\
\hline
\end{tabular}

Table 5a: Percentage occurrence of bacteria isolated from Cocoa pods. Key: +: Present; -: Absent.

Comparative distribution and occurrence of fungi isolated from Cocoa pods

The total number of fungal species isolated from each of the samples collected were presented in table $5 \mathrm{~b}$, the occurrence of each of the fungal isolates from both the infected and the uninfected as well as the percentage occurrences were also presented.

\section{Susceptibility pattern of Gram negative bacteria}

All the isolated bacteria were resistant to Septrin, Chloramphenicol, Amoxicillin, Augmentin with zone $(0 \mathrm{~mm}-7 \mathrm{~mm})$ and Pseudomonas species gave intermediate resistance to Streptomycin and Tarivid with zone $(8 \mathrm{~mm}$ $-10 \mathrm{~mm}$ ) as revealed in table 6 . On the contrary, Pseudomonas aeruginosa, Citrobacter freundii, Klebsiella oxytoca, Proteus mirabilis, Pseudomonas spp. were all susceptible to Ciprofloxacin. Citrobacter freundii, Klebsiella oxy toca, Proteus mirabilis and Pseudomonas spp. also displayed susceptibility to Pefloxacin. Then, Citrobacter freundii, Klebsiella oxytoca, Proteus mirabilis showed susceptibility to Tarivid whereas only, Pseudomonas aeruginosa Klebsiella oxytoca, Proteus mirabilis were susceptible to Sparfloxacin. 


\begin{tabular}{|l|c|c|c|c|c|c|c|c|}
\hline \multicolumn{1}{|c|}{ Isolates } & \multicolumn{3}{c|}{ Infected } & \multicolumn{3}{c|}{ Uninfected } & Total & Occurrence \\
\hline \multicolumn{1}{|c}{ Fungal isolates } & A & B & C & A & B & C & & (\%) \\
\hline Aspergillus aculeatinus & + & - & - & & - & - & 1 & 5 \\
\hline Penicillium chrysogenum & + & - & - & & - & - & 1 & 5 \\
\hline Chloridium chlamydosporis & & + & - & & - & - & 1 & 5 \\
\hline Aspergillus niger & & - & + & & - & - & 1 & 5 \\
\hline Saccharomycodes ludwigii & & + & + & & - & - & 2 & 10 \\
\hline Phytophthora palmivora & + & - & - & & - & - & 1 & 5 \\
\hline Aspergillus flavus & & - & - & + & - & - & 1 & 5 \\
\hline Saccharomyces cerevisiae & & + & - & & + & - & 2 & 10 \\
\hline Aspergillus tamari & & - & - & & - & + & 1 & 5 \\
\hline Fusarium sp. & + & + & + & + & + & + & 6 & 30 \\
\hline Penicillium camemberti & + & - & + & - & - & - & 2 & 10 \\
\hline Alternaria sp. & - & + & - & - & - & - & 1 & 5 \\
\hline Total & 5 & 5 & 4 & 2 & 2 & 2 & 20 & 100 \\
\hline
\end{tabular}

Table 5b: Percentage occurrence of fungi isolated from Cocoa pods

Key: +: Present; -: Absent

\begin{tabular}{|c|c|c|c|c|c|c|c|c|c|c|}
\hline $\begin{array}{l}\text { Isolate } \\
\text { code }\end{array}$ & OF) & g) & & & & g) & g) & g) & CN & PE \\
\hline $\mathrm{I}_{1} \mathrm{~b}$ & & 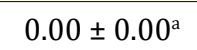 & & $1^{\mathrm{b}}$ & $32^{\mathrm{b}}$ & $2^{\mathrm{b}}$ & $0^{a}$ & $0^{\mathrm{a}}$ & $j^{a}$ & J \\
\hline $\mathrm{I}_{2} \mathrm{a}$ & & & & & & & & $0^{\mathrm{a}}$ & & \\
\hline $\mathrm{I}_{2} \mathrm{c}$ & $0^{\mathrm{a}}$ & $0.00 \pm$ & $.00^{\mathrm{a}}$ & 0.00 & $00^{\mathrm{a}}$ & $00^{\mathrm{a}}$ & 0.00 & $.00 \pm 0.00^{\mathrm{a}}$ & $0.00 \pm 0.00^{\mathrm{a}}$ & $0.00^{\mathrm{a}}$ \\
\hline $\mathrm{I}_{3} \mathrm{a}$ & & & & & & & & & & \\
\hline $\mathrm{I}_{3} \mathrm{~b}$ & $00^{\mathrm{a}}$ & $00^{\mathrm{a}}$ & $00^{\mathrm{a}}$ & $0.0 c$ & $00^{\mathrm{a}}$ & $00^{\mathrm{a}}$ & 0.00 & $0.00 \pm 0.00^{\mathrm{a}}$ & $0.00=$ & 0.00 \\
\hline $\mathrm{I}_{3} \mathrm{c}$ & & $b^{a}$ & & & & & $.00^{\mathrm{a}}$ & $.00^{\mathrm{a}}$ & $00^{\mathrm{a}}$ & $.00^{\mathrm{a}}$ \\
\hline $\mathrm{UI}_{1} \mathrm{a}$ & $.00 \pm 0.23^{c}$ & $12.00 \pm 0.21^{\mathrm{c}}$ & $7.02 \pm 0.01^{\mathrm{b}}$ & $0.00 \pm 0.00^{\mathrm{a}}$ & $12.00 \pm 0.44^{\mathrm{b}}$ & $14.00 \pm 0.43^{\mathrm{c}}$ & $0.00 \pm 0.00^{\mathrm{a}}$ & $0.00 \pm 0.00^{\mathrm{a}}$ & $0.00 \pm 0.00^{\mathrm{a}}$ & $12.02 \pm 0.36^{\mathrm{b}}$ \\
\hline $\mathrm{UI}_{2} \mathrm{a}$ & $11^{\mathrm{c}}$ & 0.00 & $00^{a}$ & 6.03 & $12.00 \pm 0.16^{b}$ & 12.00 & 0.00 & $0.00=$ & $7.00 \pm 0.52^{\mathrm{b}}$ & $12.00 \pm 0.01^{b}$ \\
\hline $\mathrm{UI}_{3} \mathrm{a}$ & $0.00 \pm 0.15^{\mathrm{b}}$ & $0.00 \pm 0.00^{\mathrm{a}}$ & $.00 \pm 0.00^{\mathrm{a}}$ & $7.00 \pm 0.18^{\mathrm{b}}$ & $0.00 \pm 0.00^{\mathrm{a}}$ & $12.00 \pm 0.19^{\mathrm{b}}$ & $7.20 \pm 0.22^{\mathrm{b}}$ & $6.00 \pm 0.91^{b}$ & $7.10 \pm 0.58^{\mathrm{b}}$ & $12.00 \pm 0.00^{\mathrm{b}}$ \\
\hline
\end{tabular}

Table 6: Susceptibility pattern of Gram negative bacteria.

Values are presented as mean \pm SE and values in the same column carrying same superscript are not significantly different using Duncan's new multiple range test at $\mathrm{p} \leq 0.05$

Key: $\mathrm{I}_{1} \mathrm{~b}$ : Pseudomonas aeruginosa; $\mathrm{I}_{2} \mathrm{a}$ : Serratia fonticola; $\mathrm{I}_{2} \mathrm{c}$ : Proteus penneri; $\mathrm{I}_{3} \mathrm{a}$ : Enterobacter cloacae; $\mathrm{I}_{3} \mathrm{~b}$ : Citrobacter freundii; $\mathrm{I}_{3} \mathrm{c}$ : Edwardsiella tarda; $\mathrm{UI}_{1} \mathrm{a}$ Klebsiella oxytoca; UI a: Proteus mirabilis; UI a: Pseudomonas species; OFX: Tarivid; S: Streptomycin; SXT: Septrin; CH: Chloramphenicol; SP: Sparfloxacin;

CPX: Ciprofloxacin; AM: Amoxicillin; AU: Augmentin; CN: Gentamycin; PEF: Pefloxacin

Susceptibility pattern of Gram positive bacteria

Bacillus subtilis revealed high susceptibility to Ciprofloxacin, Rocephin, $\mathrm{Pe}$ floxacin and Erythromycin, while Bacillus species showed high susceptibility to Rocephin only as seen in table 7. On the other hand, Bacillus species were resistant to the remaining antibiotics.

Antifungal susceptibility of fungal isolates

The result in table 8 revealed the susceptibility of fungal isolates to commercial antifungal agents. Only Phytophthora palmivora was susceptible to Griseofulvin while the other fungi were not susceptible; only Penicillium camember was not susceptible to Ketoconazole, while all the other fungi were susceptible; Penicillium chrysogenum, Penicillium camemberti, Alternaria species were not susceptible to Itraconazole while other fungi were susceptible; Alternaria species, Aspergillus niger, Saccharomyces cerevisiae were not susceptible to Clotrimazole while others were susceptible; DMSO is the negative control.

Antibiotic activity of abdominal gland extract of $O$. longinoda

All the bacterial isolates were susceptible to both the extract and Ciprofloxacin except Klebsiella oxytoca which was not susceptible to Ciprofloxacin as revealed in table 9 .

\begin{tabular}{|l|c|c|c|c|c|c|c|c|c|c|}
\hline $\begin{array}{c}\text { Isolate } \\
\text { Code }\end{array}$ & $\mathbf{S}(\mathbf{3 0} \boldsymbol{\mu \mathbf { g }})$ & $\mathbf{C P X}(\mathbf{1 0} \boldsymbol{\mu g})$ & $\mathbf{R}(\mathbf{2 5} \boldsymbol{\mu \mathbf { g }})$ & $\mathbf{A M}(\mathbf{3 0} \boldsymbol{\mu g})$ & $\mathbf{Z ~ ( 2 0} \boldsymbol{\mu g})$ & $\mathbf{A P X}(\mathbf{3 0} \boldsymbol{\mu g})$ & $\mathbf{C N}(\mathbf{1 0} \boldsymbol{\mu g})$ & PEF $(\mathbf{1 0} \boldsymbol{\mu g})$ & $\mathbf{E}(\mathbf{1 0} \boldsymbol{\mu g})$ & $\begin{array}{c}\text { SXT } \\
(\mathbf{3 0} \boldsymbol{\mu g})\end{array}$ \\
\hline $\mathrm{I}_{1} \mathrm{a}$ & $0.00 \pm 0.00^{\mathrm{a}}$ & $17.00 \pm 0.21^{\mathrm{b}}$ & $12.00 \pm 0.21^{\mathrm{b}}$ & $7.01 \pm 0.42^{\mathrm{a}}$ & $0.00 \pm 0.00^{\mathrm{a}}$ & $0.00 \pm 0.00^{\mathrm{a}}$ & $0.00 \pm 0.00^{\mathrm{a}}$ & $14.01 \pm 0.31^{\mathrm{b}}$ & $12.20 \pm 0.22^{\mathrm{b}}$ & $0.00 \pm 0.00^{\mathrm{a}}$ \\
\hline $\mathrm{I}_{2} \mathrm{~b}$ & $6.00 \pm 0.21^{\mathrm{b}}$ & $7.02 \pm 0.01^{\mathrm{a}}$ & $22.00 \pm 0.00^{\mathrm{b}}$ & $0.00 \pm 0.00^{\mathrm{a}}$ & $0.00 \pm 0.00^{\mathrm{a}}$ & $0.00 \pm 0.00^{\mathrm{a}}$ & $0.00 \pm 0.00^{\mathrm{a}}$ & $0.00 \pm 0.00^{\mathrm{a}}$ & $0.00 \pm 0.00^{\mathrm{a}}$ & $0.00 \pm 0.00^{\mathrm{a}}$ \\
\hline
\end{tabular}

Table 7: Susceptibility pattern of Gram positive bacteria.

Values are presented as mean \pm SE and values in the same column carrying same superscript are not significantly different using Duncan's new multiple range test at $\mathrm{p} \leq 0.05$

Key: I a: Bacillus subtilis; I $\mathrm{I}_{2} \mathrm{~b}$ : Bacillus species; S: Streptomycin; CPX: Ciprofloxacin; R: Rocephin; AM: Amoxicillin; Z: Zinacef; APX: Ampiclox; CN: Gentamycin; PEF: Pefloxacin; E: Erythromycin; SXT: Septrin.

\begin{tabular}{|l|c|c|c|c|c|}
\hline \multicolumn{1}{|c|}{ Fungal Isolate } & $\mathbf{A}(\mathbf{m m})$ & $\mathbf{B}(\mathbf{m m})$ & $\mathbf{C}(\mathbf{m m})$ & $\mathbf{D}(\mathbf{m m})$ & $\mathbf{E}(\mathbf{m m})$ \\
\hline Aspergillus aculeatinus & $0.00 \pm 0.00^{\mathrm{a}}$ & $20.68 \pm 0.17^{\mathrm{e}}$ & $12.03 \pm 0.26^{\mathrm{b}}$ & $24.00 \pm 0.35^{\mathrm{f}}$ & $0.00 \pm 0.00^{\mathrm{a}}$ \\
\hline Penicillium chrysogenum & $0.00 \pm 0.00^{\mathrm{a}}$ & $11.97 \pm 0.55^{\mathrm{b}}$ & $0.00 \pm 0.00^{\mathrm{a}}$ & $24.00 \pm 0.00^{\mathrm{f}}$ & $0.00 \pm 0.00^{\mathrm{a}}$ \\
\hline Penicillium camemberti & $0.00 \pm 0.00^{\mathrm{a}}$ & $0.00 \pm 0.00^{\mathrm{a}}$ & $0.00 \pm 0.00^{\mathrm{a}}$ & $24.03 \pm 0.51^{\mathrm{f}}$ & $0.00 \pm 0.00^{\mathrm{a}}$ \\
\hline Alternaria sp & $0.00 \pm 0.00^{\mathrm{a}}$ & $34.00 \pm 0.23^{\mathrm{h}}$ & $0.00 \pm 0.00^{\mathrm{a}}$ & $0.00 \pm 0.00^{\mathrm{a}}$ & $0.00 \pm 0.00^{\mathrm{a}}$ \\
\hline Chloridium chlamydosporis & $0.00 \pm 0.00^{\mathrm{a}}$ & $24.00 \pm 0.11^{\mathrm{g}}$ & $29.00 \pm 0.05$ & $9.00 \pm 0.51^{\mathrm{b}}$ & $0.00 \pm 0.00^{\mathrm{a}}$ \\
\hline Aspergillus niger & $0.00 \pm 0.00^{\mathrm{a}}$ & $16.03 \pm 0.38^{\mathrm{c}}$ & $16.03 \pm 0.54^{\mathrm{c}}$ & $0.00 \pm 0.00^{\mathrm{a}}$ & $0.00 \pm 0.00^{\mathrm{a}}$ \\
\hline Saccharomycodes ludwigii & $0.00 \pm 0.00^{\mathrm{a}}$ & $24.00 \pm 0.00^{\mathrm{g}}$ & $24.00 \pm 0.03^{\mathrm{g}}$ & $19.03 \pm 0.61^{\mathrm{e}}$ & $0.00 \pm 0.00^{\mathrm{a}}$ \\
\hline Phytophthora palmivora & $13.96 \pm 0.54^{\mathrm{b}}$ & $24.06 \pm 0.18^{\mathrm{g}}$ & $24.00 \pm 0.05^{\mathrm{g}}$ & $34.00 \pm 0.58^{\mathrm{g}}$ & $0.00 \pm 0.00^{\mathrm{a}}$ \\
\hline Aspergillus flavus & $0.00 \pm 0.00^{\mathrm{a}}$ & $22.97 \pm 0.43^{\mathrm{f}}$ & $23.00 \pm 0.00^{\mathrm{f}}$ & $24.03 \pm 0.19^{\mathrm{f}}$ & $0.00 \pm 0.00^{\mathrm{a}}$ \\
\hline Saccharomyces cerevisiae & $0.00 \pm 0.00^{\mathrm{a}}$ & $20.00 \pm 0.05^{\mathrm{e}}$ & $20.03 \pm 0.54^{\mathrm{d}}$ & $0.00 \pm 0.00^{\mathrm{a}}$ & $0.00 \pm 0.00^{\mathrm{a}}$ \\
\hline Aspergillus tamari & $0.00 \pm 0.00^{\mathrm{a}}$ & $24.00 \pm 0.58^{\mathrm{g}}$ & $23.96 \pm 0.55^{\mathrm{f}}$ & $16.00 \pm 0.41^{\mathrm{d}}$ & $0.00 \pm 0.00^{\mathrm{a}}$ \\
\hline Fusarium sp & $0.00 \pm 0.00^{\mathrm{a}}$ & $19.00 \pm 0.00^{\mathrm{d}}$ & $12.03 \pm 0.04^{\mathrm{b}}$ & $12.00 \pm 0.58^{\mathrm{c}}$ & $0.00 \pm 0.00^{\mathrm{a}}$ \\
\hline
\end{tabular}

Table 8: Antifungal Sensitivity Test.

Values are presented as mean \pm SE and values in the same column carrying same superscript are not significantly different using Duncan's new multiple range test at $\mathrm{p} \leq 0.05$

Key: A: Griseofulvin; B: Ketoconazole; C: Itraconazole; D: Clotrimazole; E: DMSO 


\begin{tabular}{|l|c|c|c|c|}
\hline \multicolumn{1}{|c|}{ Bacterial Isolate } & A (mm) & B (mm) & C (mm) & D $(\mathbf{m m})$ \\
\hline Pseudomonas aeruginosa & $14.00 \pm 0.29^{\mathrm{d}}$ & $14.00 \pm 0.00^{\mathrm{de}}$ & $14.00 \pm 0.00^{\mathrm{d}}$ & $16.00 \pm 0.46^{\mathrm{d}}$ \\
\hline Serratia fonticola & $9.00 \pm 0.23^{\mathrm{b}}$ & $12.00 \pm 0.55^{\mathrm{d}}$ & $13.00 \pm 0.00^{\mathrm{c}}$ & $16.00 \pm 0.00^{\mathrm{d}}$ \\
\hline Bacillus subtilis & $19.00 \pm 0.06^{\mathrm{e}}$ & $13.03 \pm 0.06^{\mathrm{f}}$ & $14.00 \pm 0.29^{\mathrm{d}}$ & $19.00 \pm 0.23^{\mathrm{b}}$ \\
\hline Proteus penneri & $11.03 \pm 0.04^{\mathrm{c}}$ & $10.00 \pm 0.58^{\mathrm{c}}$ & $12.00 \pm 0.00^{\mathrm{c}}$ & $20.00 \pm 0.00^{\mathrm{f}}$ \\
\hline Enterobacter cloacae & $9.03 \pm 0.50^{\mathrm{b}}$ & $9.00 \pm 0.58^{\mathrm{de}}$ & $11.00 \pm 0.12^{\mathrm{b}}$ & $14.01 \pm 0.46^{\mathrm{b}}$ \\
\hline Citrobacter freundii & $14.00 \pm 0.00$ & $9.00 \pm 0.17^{\mathrm{de}}$ & $12.03 \pm 0.06^{\mathrm{b}}$ & $14.00 \pm 0.00^{\mathrm{b}}$ \\
\hline Edwardsiella tarda & $11.00 \pm 0.01^{\mathrm{c}}$ & $14.33 \pm 0.44^{\mathrm{e}}$ & $14.03 \pm 0.08$ & $19.00 \pm 0.56$ \\
\hline Klebsiella oxytoca & $0.00 \pm 0.00^{\mathrm{a}}$ & $10.00 \pm 0.06^{\mathrm{c}}$ & $12.00 \pm 0.00^{\mathrm{c}}$ & $15.00 \pm 0.00^{\mathrm{c}}$ \\
\hline Proteus mirabilis & $9.00 \pm 0.00^{\mathrm{b}}$ & $5.00 \pm 0.56^{\mathrm{b}}$ & $15.03 \pm 0.00^{\mathrm{e}}$ & $21.03 \pm 0.09^{\mathrm{g}}$ \\
\hline
\end{tabular}

Table 9: Antibiotic activity of abdominal gland extract of 0 . longinoda.

Values are presented as mean \pm SE and values in the same column carrying same superscript are not significantly different using Duncan's new multiple range test at $\mathrm{p} \leq 0.05$.

Antibacterial activity of abdominal gland extract of 0 . longinoda. B, C and D - varying concentrations of abdominal gland extract. Concentration $\mathrm{B}$ is gland from three ants; concentration $\mathrm{C}$ is gland from six ants; concentration $\mathrm{D}$, gland from twelve ants. A is, Ciprofloxacin std $100 \mu \mathrm{l}(40 \mathrm{mg} / \mathrm{ml})$.

\section{Antifungal activity of abdominal gland extract of $O$. longinoda}

All the fungal isolates were susceptible to the extract except for Penicillium chrysogenum, Penicillium camemberti, Saccharomycodes ludwigii, Phytophthora palmivora and Fusarium sp. which were not susceptible; of all the twelve isolated fungi tested with Ketoconazole, eleven (Aspergillus aculeatinus, Alternaria species, Chloridium chlamydosporis, Aspergillus niger, Phytophthora palmivora, Aspergillus flavus, Aspergillus tamari, Fusarium sp., Saccharomyces cerevisiae, Saccharomycodes ludwigii, Penicillium chrysogenum) were susceptible while the only one that was not susceptible is Penicillium camemberti.

\begin{tabular}{|l|c|c|c|c|}
\hline $\begin{array}{l}\text { Fungal } \\
\text { Isolate } \\
\text { (code is to } \\
\text { be removed) }\end{array}$ & $\mathbf{A}(\mathbf{m m})$ & $\mathbf{B}(\mathbf{m m})$ & $\mathbf{C}(\mathbf{m m})$ & $\mathbf{D}(\mathbf{m m})$ \\
\hline $\begin{array}{l}\text { Aspergillus } \\
\text { aculeatinus }\end{array}$ & $19.05 \pm 0.14^{\mathrm{b}}$ & $8.95 \pm 0.10^{\mathrm{c}}$ & $10.00 \pm 0.01^{\mathrm{b}}$ & $11.00 \pm 0.00^{\mathrm{c}}$ \\
\hline $\begin{array}{l}\text { Penicillium } \\
\text { chrysogenum }\end{array}$ & $11.90 \pm 0.15^{\mathrm{d}}$ & $0.00 \pm 0.00^{\mathrm{a}}$ & $0.00 \pm 0.00^{\mathrm{a}}$ & $0.00 \pm 0.00^{\mathrm{a}}$ \\
\hline $\begin{array}{l}\text { Penicillium } \\
\text { camemberti }\end{array}$ & $0.00 \pm 0.00^{\mathrm{a}}$ & $0.00 \pm 0.00^{\mathrm{a}}$ & $0.00 \pm 0.00^{\mathrm{a}}$ & $0.00 \pm 0.00^{\mathrm{a}}$ \\
\hline Alternaria sp. & $24.33 \pm 0.30^{\mathrm{c}}$ & $8.00 \pm 0.22^{\mathrm{d}}$ & $10.03 \pm 0.00^{\mathrm{b}}$ & $12.51 \pm 0.21^{\mathrm{c}}$ \\
\hline $\begin{array}{l}\text { Chloridium } \\
\text { chlamydo- } \\
\text { sporis }\end{array}$ & $24.36 \pm 0.05^{\mathrm{c}}$ & $6.00 \pm 0.32^{\mathrm{b}}$ & $7.90 \pm 0.03^{\mathrm{b}}$ & $9.22 \pm 0.25^{\mathrm{b}}$ \\
\hline $\begin{array}{l}\text { Aspergillus } \\
\text { niger }\end{array}$ & $24.00 \pm 0.03^{\mathrm{c}}$ & $8.34 \pm 0.15^{\mathrm{b}}$ & $9.17 \pm 0.33$ & $10.12 \pm 0.22^{\mathrm{b}}$ \\
\hline $\begin{array}{l}\text { Saccharomy- } \\
\text { codes ludwigii }\end{array}$ & $24.00 \pm 0.03^{\mathrm{c}}$ & $0.00 \pm 0.00^{\mathrm{a}}$ & $0.00 \pm 0.00^{\mathrm{a}}$ & $0.00 \pm 0.00^{\mathrm{a}}$ \\
\hline $\begin{array}{l}\text { Phytophthora } \\
\text { palmivora }\end{array}$ & $24.33 \pm 0.30^{\mathrm{c}}$ & $0.00 \pm 0.00^{\mathrm{a}}$ & $0.00 \pm 0.00^{\mathrm{a}}$ & $0.00 \pm 0.00^{\mathrm{a}}$ \\
\hline $\begin{array}{l}\text { Aspergillus } \\
\text { flavus }\end{array}$ & $20.02 \pm 0.31^{\mathrm{b}}$ & $9.33 \pm 0.00^{\mathrm{d}}$ & $12.11 \pm 0.31^{\mathrm{b}}$ & $14.89 \pm 0.06^{\mathrm{e}}$ \\
\hline $\begin{array}{l}\text { Saccharomy- } \\
\text { ces cerevisiae }\end{array}$ & $20.00 \pm 0.30^{\mathrm{b}}$ & $11.16 \pm$ & $11.17 \pm 0.06^{\mathrm{c}}$ & $12.12 \pm 0.16^{\mathrm{d}}$ \\
\hline $\begin{array}{l}\text { Aspergillus } \\
\text { tamari }\end{array}$ & $24.01 \pm 0.11^{\mathrm{c}}$ & $9.04 \pm 0.06^{\mathrm{c}}$ & $11.00 \pm 0.02^{\mathrm{b}}$ & $14.04 \pm 0.01^{\mathrm{e}}$ \\
\hline Fusarium sp. & $19.09 \pm 0.15^{\mathrm{b}}$ & $0.00 \pm 0.00^{\mathrm{a}}$ & $0.00 \pm 0.00^{\mathrm{a}}$ & $0.00 \pm 0.00^{\mathrm{a}}$ \\
\hline
\end{tabular}

Table 10: Antifungal activity of abdominal gland extract of $O$. longinoda. Values are presented as mean \pm SE and values in the same column carrying same superscript are not significantly different using Duncan's new multiple range test at $\mathrm{p} \leq 0.05$.

Antifungal activity of abdominal gland extract of 0 . longinoda. B, C and D, Increasing concentrations of abdominal gland extract. Concentration $\mathrm{B}$ is gland from three ants; concentration $\mathrm{C}$ is glands from six ants; concentration D, gland from twelve ants. A is, Ketoconazole std $100 \mu \mathrm{l}(40 \mathrm{mg} / \mathrm{ml})$
B

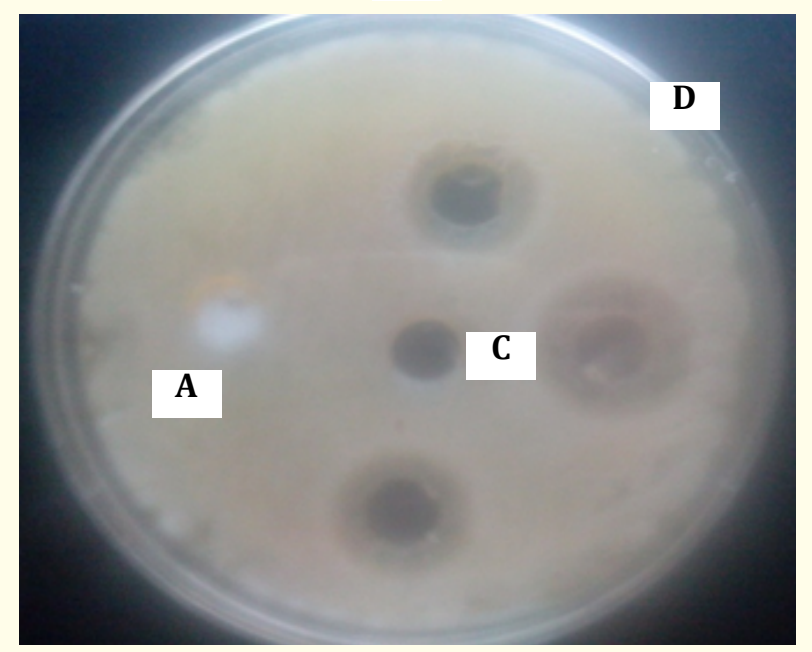

Plate 1: Antibacterial activity of Oecophylla longinoda abdominal gland extract against Klebsiella oxytoca.

Key: A: Ciprofloxacin; B: 3 Abdominal Gland Extract of 0 . longino$d a$; C: 6 Abdominal Gland Extract of 0 . longinoda ; D: 12 Abdominal Gland Extract of $O$. longinoda

C

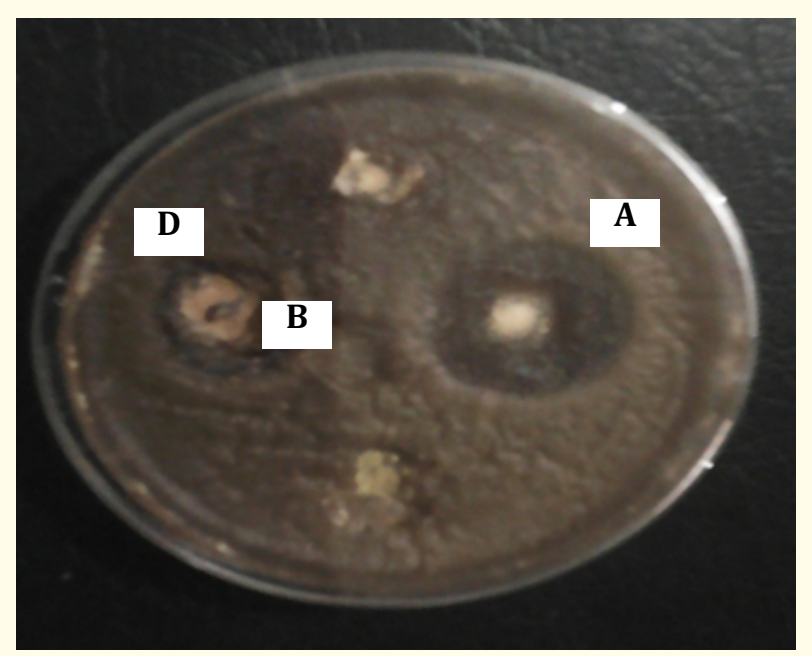

Plate 2: Antifungal activity of Oecophylla longinoda abdominal gland extract against Aspergillus niger.

Key: A: Ketaconazole; B: 3 Abdominal Gland Extract of 0 . longino$d a$; C: 6 Abdominal Gland Extract of 0 . longinoda ; D: 12 Abdominal Gland Extract of $O$. longinoda 


\section{Discussion}

These results revealed that microorganisms can be isolated from both infected and healthy Cocoa pods. The result showed that microorganisms (bacteria and fungi) isolated from Cocoa pods were more in infected pods than in healthy pods. The microorganisms isolated from the Cocoa pods is in agreement with the work of Adejumo and Adejoro [27] and Nasril [28].

Twenty-two (22) bacterial isolates were obtained of which Proteus penneri had the highest percentage occurrence of $27.3 \%$ while Serratia and Citrobacter freundii had the lowest percentage occurrence of 4.5\%. 20 fungal isolates were obtained of which Fusarium sp. had the highest percentage occurrence of 30\%, Aspergillus aculeatinus, Penicillium chrysogenum, Chloridium chlamydosporis, Phytophthora palmivora, Aspergillus flavus, Aspergillus tamari; Alternaria sp. had the lowest percentage occurrence of $5 \%$.

All the tested bacterial isolates showed increase in susceptibility as the concentration of the extract increases this is in agreement with the work of Vidhu and Evans [26]; all the isolated bacteria were susceptible to the positive control (Ciprofloxacin) except Klebsiella oxytoca which was not susceptible to Ciprofloxacin.

Ketoconazole appeared to be the most effective antifungal agent in the control group. Of all the twelve fungal isolates tested with Ketoconazole, eleven (Aspergillus aculeatinus, Alternaria sp., Chloridium chlamydosporis, Aspergillus niger, Phytophthora palmivora, Aspergillus flavus, Aspergillus tamari, Fusarium sp., Saccharomyces cerevisiae, Saccharomycodes ludwigii, Penicillium chrysogenum) were susceptible while (Penicillium camemberti) was not susceptible.

All the fungal isolates showed increase in susceptibility as the concentration of the extract increases, this is in correspondence with the work of Vidhu and Evans [26] except for Penicillium chrysogenum, Penicillium camemberti, Saccharomycodes ludwigii, Phytophthora palmivora and Fusarium species which were not susceptible to all the concentrations; hence, in order to protect cocoa pods from infections, the population of $O$. longinoda must be very abundant, which was also suggested by Vanderplank [29].

\section{Conclusion and Recommendation}

The isolation of microorganisms from cocoa pods showed that both bacteria and fungi are present on both diseased and healthy pods and that the fact that microorganisms were isolated from both healthy and infected pods does not mean that they are both infected. On the other hand, the healthy pods might have been infected but have not started manifesting the symptoms of diseases. Oecophylla longinoda is a very good biocontrol agent as well as a potent antimicrobial agent which can be used by farmers in place of pesticides because it is relatively cheaper than pesticides and environmentally friendly as it is not harmful to human and the environment.

\section{Bibliography}

1. Acebo-Guerrero Y., et al. "Management of black pod rot in Cacao (L.): A review". Fruits 67.1 (2012): 41-48.

2. Iremiren G O. "Soil fertility and ageing cocoa farms in Nigeria". WCF Partnership Meeting and Round Table Session, Accra 16 (2011): 26-27.
3. Gorenz A M and Okaisabor E K. "Phytophthora pod rot disease in Nigeria. In progress in Tree Crop Research in Nigeria ( $2^{\text {nd }}$ Edition) Cocoa Research Institute Nigeria Ibadan (1971): 124-133.

4. Bowers J H., et al. "The Impact of Plant Diseases on World Chocolate Prod". Plant Health Progress (2001).

5. Maddison A C and Ward M R. "The International Black Pod Projects: 1979 Review". Proceedings of $7^{\text {th }}$ International. Cacao Research. Conference Douala, Cameroon (1981): 261-266.

6. Gregory P H. "Speculations on Phytophthora as a cacao root pathogen". Proceedings of $7^{\text {th }}$ International. Cacao Research. Conference Douala, Cameroon (1981): 267-269.

7. Purdy L H. "Fungal diseases of cacao. In: Research methodology in biocontrol of Plant diseases with special reference to fungal diseases of cocoa". Workshop Manual CATIE, Tirrialba, Costa Ric. Eds : U Krauss, P Hebbar (1999): 7-18.

8. Opoku I Y., et al. "Phytophthora megakarya: a potential threat to the cocoa industry in Ghana?" Ghana Journal of Agricultural Science 33.2 (2000): 237-248.

9. Friedrich T. "Agricultural Pesticide Application. FAO Agricultural Engineering Branch AGSE". Food and Agriculture Organization of the United Nations, Rome (1996).

10. Meijden G Van der. "Pesticide Application Techniques in West Africa". A study by the Agricultural Engineering Branch of FAO through the FAO Regional Office for Africa 17 (1998).

11. Way M J and Khoo K C. "Role of ants in pest management". Annual Review of Entomology 37 (1992): 479-503.

12. Van Mele P. "A historical review of research on the weaver ant Oecophylla in biological control". Agriculture for Entomology 10 (2008): 13-22.

13. Peng R K and Christian K. "Effective control of jarvis's fruit fly, Bactrocera jarvisi (diptera: Tephritidae), by the weaver ant, Oecophylla smaragdina (hymenoptera: Formicidae), in mango orchards in the northern territory of Australia". International Journal of Pest Management 52.4 (2006): 275282.

14. Peng R K., et al. "Implementing ant technology in commercial cashew plantations and continuation of transplanted green ant colony monitoring. W04/088. Australian Government". Rural Industries Research and Development Corporation (2004).

15. Peng R K and Christian K. "Integrated pest management in mango orchards in the Northern Territory Australia, using the weaver ant, Oecophylla smaragdina (Hymenoptera : Formicidae), as a key element". International Journal of Pest Management 51.2 (2005b): 149-155.

16. Dejean A. "Orcadian rhythm of Oecophylla longinoda in relation to territoriality and predatory behavior". Physiology Entomology 15.4 (1990): 393-403.

17. Vayssieres J F., et al. "Field observational studies on circadian activity pattern of Oecophylla longinoda (latreille) (hymenoptera: Formicidae) in relation to abiotic factors and mango cultivars". International Journal of Biological Chemical Science 5.2 (2011): 790-802. 
18. Dejean A. "Adaptation of Oecophylla longinoda to spatiotemporal variations in prey density". Entomophaga 36 (1991): 29-54.

19. Chen Y and Alue R D. "Ants used as food and medicine in China". The Food Insects Newsletter 7 (1994): 1-10.

20. Oudhia P. “Traditional medicinal knowledge about red ant Oecophylla smaragdina (Fab.)" [Hymenoptera; Formicidae] in Chhattisgarh, India". Insect Environment 8 (2002): 114-115.

21. Arnold B. "Fungal endophytes of tropical trees: Methods and potential for biological control of fungal pathogens of cocoa. Research methodology in biocontrol of plant diseases with special reference to fungal diseases of cocoa". Worshop manual, CATIE, Turrialba, Costa Rica Edited by Ulrike Krauss and Prakash Hebbar (1999): 44 -54.

22. Evans H C., et al. "Endophytes and mycoparasites associated with an indigenous forest tree, Theobroma gileri, in Ecuador and preliminary assessment of their potential as biocontrol agents of cocoa diseases". Mycological Progress 2.2 (2003): 149-160.

23. Rubini M R., et al. "Diversity of endophytic fungal community of cacao (Theobroma cacao L.) and biological control of Crinipellis perniciosa, causal agent of Witches' Broom Disease". International Journal of Biological Science 1.1 (2005): 24-33.

24. Fawole M O and Oso B A. "Characterization of bacteria: Laboratory Manual of Microbiology". $4^{\text {th }}$ Edition, Spectrum Books Limited, Ibadan, Nigeria (2007): 24-33.

25. Cheesbrough M. "District Laboratory Practice in Tropical Countries". $2^{\text {nd }}$ Edition, Cambridge University Press, Cambridge, UK (2006): 165-176.

26. Vidhu V V and Evans D A. "Ethnoentomological values of Oecophylla". Current Science 109.3 (2015): 573.

27. Adejumo T 0 and Adejoro D O. "Assessment of bacteria, fungi and protozoa in three Theobroma cacao soils". International Journal of Sciences 4 (2015): $30-32$.

28. Nasril N. "Black pod disease caused by Phytophthora palmivora in assigned center production in West Sumatra Indonesia". Research Journal of Pharmaceutical Biological and Chemical Sciences 7.4 (2016): 1759-1760.

29. Vanderplank F L. "The bionomics and ecology of the red tree ant, Oecophylla species and its relationship to the coconut bug Pseudotheraptus wayi Brown (Coreidae)". Journal of Animal Ecology 29 (1960): 15-33

\section{Volume 1 Issue 3 March 2018}

(C) All rights are reserved by Oladosu Tolulope 0. , et al. 